\title{
Clinical Outcomes of Unicompartmental Knee Arthroplasty in Patients with Full- Versus Partial-Thickness Cartilage Loss: A Systematic Review and Meta-Analysis
}

\author{
Jinhui Ma \\ China-Japan Friendship Hospital \\ Yan Yan \\ China-Japan Friendship Hospital \\ Weiguo Wang \\ China-Japan Friendship Hospital \\ Bailiang Wang ( $\square$ Wang_orthopaedic@126.com) \\ China-Japan Friendship Hospital \\ Debo Yue \\ China-Japan Friendship Hospital \\ Wanshou Guo \\ China-Japan Friendship Hospital
}

\section{Research article}

Keywords: Unicompartmental knee arthroplasty, full-thickness cartilage loss, partial-thickness cartilage loss, postoperative outcome, reoperation rate Posted Date: November 3rd, 2020

DOl: https://doi.org/10.21203/rs.3.rs-99413/v1

License: (c) (i) This work is licensed under a Creative Commons Attribution 4.0 International License. Read Full License 


\section{Abstract}

Bakground This meta-analysis was designed to compare postoperative patient-reported outcomes and reoperation rates following unicompartmental knee arthroplasty (UKA) between patients with full-thickness cartilage loss (FTCL) and partial-thickness cartilage loss (PTCL).

Methods Multiple databases, including PubMed, Embase, Cochrane Library, and CNKI, were searched to identify studies comparing the Oxford Knee Score (OKS), American Knee Society (AKS) score, and reoperation rates between patients with FTCL and PTCL following UKA. Mean differences were calculated for continuous outcomes, and odds ratios were calculated for binary outcomes.

Results A total of 613 UKAs (233 PTCL and 380 FTCL) from five retrospective cohort studies were included. Three studies compared OKSs after $\geq 2$ years of follow-up. The mean difference in postoperative OKSs was significantly higher by 2.92 in the FTCL patients than in the PTCL patients ( $95 \%$ confidence interval $[\mathrm{Cl}]-5.29$ to $-0.55 ; \mathrm{P}=0.02)$. The improvement in the OKS was significantly higher by 2.69 in the FTCL patients than in the PTCL patients ( $95 \%$ $\mathrm{Cl}-4.79$ to $-0.60 ; \mathrm{P}=0.01$ ). AKS-knee and AKS-function scores were assessed in two studies at a mean follow-up time of $>3.9$ years. The mean difference in AKS-knee scores was similar between the groups $(95 \% \mathrm{Cl}-9.14$ to $-3.34 ; \mathrm{P}=0.36)$, whereas the pooled mean difference in AKS-function scores was 5.63 higher in the FTCL group $(95 \% \mathrm{Cl}-9.27$ to $-1.98 ; \mathrm{P}=0.002)$. All studies presented data on reoperation rates and showed that they were statistically higher in patients who underwent UKA with PTCL (OR 2.24, 95\% Cl 1.15-4.38, P = 0.02).

Conclusions Compared with PTCL, FTCL achieved superior postoperative patient-reported outcomes and lower reoperation rates following UKA.

\section{Bakground}

As one of the most common diseases worldwide, knee osteoarthritis (KOA) is a leading cause of disability and decreasing the quality of life in older adults. Given that the proportions of obese and older adults have been increasing rapidly, the prevalence of KOA has also increased to levels not previously seen. In China, $18 \%$ of the population suffers from KOA, and $>70 \%$ of older adults ( $>60$ years old) showed different degrees of KOA. As a result, the social burden caused by KOA has been increasing [1, 2]. So far, plenty of methods have been proposed to either prevent or treat different stages of KOA, such as functional exercise, avoiding weight-bearing, oral non-steroidal drugs, Chinese herbal medicine, joint injection, arthroscopy, knee osteotomy, and joint replacement.

Among all of the methods mentioned above, unicompartmental knee arthroplasty (UKA) is a feasible choice for treating KOA and spontaneous osteonecrosis of the knee (SONK) [3-6]. Given that patients with partial-thickness cartilage loss (PTCL) seem to have inferior postoperative patientreported outcomes and higher reoperation rates, we usually recommend performing UKA on patients with full-thickness cartilage loss (FTCL) in the medial compartment of the knee, which is a condition causing "bone-to-bone" abrasion, as shown by obliteration of the joint space on a standing anterior-posterior radiograph, [7]. Goodfellow stated that compared with FTCL patients, PTCL patients have variable outcomes, and many do not benefit from the operation in the short term $[7,8]$. However, that assertion was not based on evidence. Some surgeons have also performed UKA on KOA patients with PTCL and appeared to have achieved acceptable outcomes [9].

This meta-analysis was designed to evaluate the applicability of PTCL in the medial compartment of the knee as an indication for UKA. We compared postoperative patient-reported outcomes and reoperation rates between patients undergoing UKA by FTCL and by PTCL.

\section{Methods}

This meta-analysis was conducted in accordance with the recommendations of the Cochrane Collaboration and the Quality of Reporting of Metaanalyses guidelines.

\subsection{Search Strategy}

The Preferred Reporting Items for Systematic Reviews and Meta-Analyses statement was used for this meta-analysis. Two investigators (Yan Yan and Jinhui Ma) independently searched multiple comprehensive databases, including PubMed, Embase, the Cochrane Library, and CNKI, up to October 2019 for the study and compared postoperative patient-reported outcomes and reoperation rates in patients with UKA treated with FTCL or PTCL. The following three categories of keywords (and related synonyms) were used to build a sensitive search strategy and provide a systematical review:

"unicompartmental knee arthroplasty," "cartilage loss," and "outcome." There were no restrictions on language, year of publication, or type of publication. Search terms were used in the "all fields" item to expand the literature search. We tried to use Medical Subject Headings words when searching in PubMed and combined all of the synonyms relevant to keywords by applying the Boolean command "OR" in each category. Furthermore, search terms were truncated with a "*" symbol to find all terms beginning with a specific word. After the initial electronic search, relevant articles, and their bibliographies were searched manually. Articles identified were assessed individually for inclusion.

\subsection{Inclusion Criteria and Study Selection}

All of the studies included in this meta-analysis were independently reviewed and selected by two authors according to predefined inclusion criteria. Titles and abstracts were read; if suitability could not be determined, the full article was evaluated. Studies were included in our meta-analysis if they met the following criteria: (1) already published, full text, peer-reviewed articles; (2) randomized controlled trial or cohort study; (3) studies designed to compare the postoperative patient-reported outcomes or revision rates of UKA performed on patients with various degrees of cartilage injury or joint space loss; (4) 
inclusion of at least one of the three parameters assessing postoperative outcome: the OKS, American Knee Society (AKS) score, and reoperation rate; (5) the number of patients in each group (FTCL and PTCL) and the means and standard deviations of the parameters mentioned above were fully reported; (6) use of adequate statistical methods to compare the parameters between the two groups; (7) eligible for pooling data for meta-analysis. The following exclusion criteria were applied: (1) studies without available full text and unpublished studies; (2) case-control studies, case series or reports, or reviews; (3) animal studies, cadaver studies, or laboratory studies; (4) study data that were incomplete, inconsistent, or impossible to synthesize. Two authors independently assessed the full study article to see if it met the inclusion criteria. Where there was disagreement or doubt, the full article was reviewed by two authors. Disagreements were resolved by discussion with each other. The corresponding author was consulted in cases of unresolved disagreement.

\subsection{Methodological Quality}

Two reviewers (Yan Yan and Jinhui Ma) used the Newcastle-Ottawa Quality Assessment Scale Table (NOQAS) [10], as recommended by the Cochrane Non-Randomized Studies Methods Working Group, to assess the methodological quality of each study. NOQAS consists of items related to population selection, comparability of exposed and unexposed, and adequacy of outcome assessment (including ascertainment and attribution of outcome), all of which were used to assess the quality of the included five studies. The star system of the Newcastle-Ottawa Scale, which awards stars according to the level of bias, was adjusted to a scale that included only low (one star), high (no star), and unclear bias.

\subsection{Data Extraction}

Two authors independently extracted information from all of the included studies. The following information was extracted from all eligible trials: (1) study information: author, year, country/region, and the number of references; (2) study design and time of follow-up; (3) study population: population size, cohort, age, sex, body mass index (BMI), comorbidities; (4) scoring scale used to evaluate rehabilitation; (5) definition of different outcomes; (6) confounding factors related to postoperative outcomes and rates of revision, such as sex, BMI, complications, the preoperative function of sports, surgeon implication, or other psychosocial-influencing factors.

\subsection{Statistical Analysis}

Heterogeneity among studies was evaluated by using $\mathrm{l}^{2}$ tests. If the $\mathrm{I}^{2}$ was $\leq 50 \%$, we considered the study to have low heterogeneity. A fixed-effects model was employed; otherwise, the random-effects model was used. The mean difference and $95 \%$ confidence intervals (95\% $\mathrm{Cl})$ were calculated for continuous outcomes, and odds ratios (ORs) and $95 \% \mathrm{Cl}$ were calculated for binary outcomes. A P-value of $<0.05$ was accepted as indicative of statistical significance. All of the statistical analyses were performed completely by using the Review Manager software from the Cochrane Collaboration (Version 5.3.).

\section{Results}

\subsection{Study Inclusion}

A total of 381 studies were identified in the initial search (376 through an electronic database, through other sources). After removal of duplicates, 364 records were identified, 352 of which were excluded mainly for two reasons: no direct comparison between PTCL and FTCL cases $(n=351)$ and the lack of a uniformed measurement of outcome $(n=1)$. The full text of the remaining 12 articles was then reviewed for more details, and seven of these were excluded for failing to distinguish PTCL and FTCL case with conceivable criteria. Eventually, five studies meeting the inclusion criteria were included in this meta-analysis. The flow diagram of the study selection procedure is shown in Fig. 1.

\subsection{Study Characteristics and Patient Populations}

The selected five studies included 233 knees with PTCL and 380 knees with FTCL who underwent medial compartment UKA. Three studies compared OKS scores, among which two studies also compared the AKS-knee and function scores. The reoperation rates of all five included studies were compared. The detailed sample size and measured parameters are presented in Table 1. 
Table 1

Characteristics of the included studies

\begin{tabular}{|c|c|c|c|c|c|c|c|}
\hline Study (year) & $\begin{array}{l}\text { No. } \\
\text { PTCL/FTCL }\end{array}$ & $\begin{array}{l}\text { Male } \\
\text { patients } \\
\text { (PTCL/FTCL) }\end{array}$ & $\begin{array}{l}\text { Mean age } \\
\text { (PTCL/FTCL) }\end{array}$ & $\begin{array}{l}\text { Mean follow- } \\
\text { up time }\end{array}$ & $\begin{array}{l}\text { Mean BMI } \\
\text { (PTCL/FTCL) }\end{array}$ & Outcomes & $\begin{array}{l}\text { Quality } \\
\text { score }\end{array}$ \\
\hline $\begin{array}{l}\text { Niinimäki et al. } \\
2011\end{array}$ & $18 / 39$ & unclear & 58 in average & 63 month & 30 in average & Reoperation rate & $8 \square$ \\
\hline $\begin{array}{l}\text { Pandit et al. } \\
2011\end{array}$ & $29 / 29$ & unclear & $\begin{array}{l}62(40-82) / 63 \\
(41-81)\end{array}$ & 2 year & unclear & OKS score, Reoperation rate & $7 \square$ \\
\hline $\begin{array}{l}\text { Maier et al. } \\
2015\end{array}$ & $32 / 32$ & $15 / 15$ & $\begin{array}{l}63(46-78) / 61 \\
(48-72)\end{array}$ & 3.9 year & unclear & $\begin{array}{l}\text { OKS score, AKS score,and } \\
\text { Reoperation rate }\end{array}$ & $7 \square$ \\
\hline $\begin{array}{l}\text { Berend et al. } \\
2017\end{array}$ & $60 / 92$ & $33 / 40$ & $62.0 / 62.3$ & 4.2 year & $31.6 / 30.9$ & Reoperation rate & 7ロ \\
\hline $\begin{array}{l}\text { Hamilton et al. } \\
2017\end{array}$ & $94 / 188$ & $40 / 84$ & $64.2 / 63.6$ & 6 year & $29.1 / 29.7$ & $\begin{array}{l}\text { OKS score, AKS score,and } \\
\text { Reoperation rate }\end{array}$ & $6 \square$ \\
\hline
\end{tabular}

\subsection{Quality of the Included Studies}

All five studies included in this meta-analysis showed a low risk of selection bias. Considering that a much lower number of patients underwent UKA with PTCL than UKA with FTCL [11, 12], two studies (Niinimäki 2011 [13] and Berend 2017 [14]) compared consecutive UKA cases. One study (Hamilton 2017 [15]) followed consecutive PTCL-defined UKA patients and matched them 1:2 by age, sex, and preoperative OKS with patients who had FTCL on both the femur and tibia. The other two studies (Pandit 2011 [16] and Maier 2015 [17]) reviewed a hospital database to identify patients with PTCL and created a matched control group according to sex, preoperative OKS, age at operation, and time to follow-up. However, two studies (Berend 2017[14] and Hamilton 2017 [15]) had a high risk of attrition bias because of the high proportion of patients lost to follow-up (13\% and $44 \%$ in the second year, respectively). All five studies were thought to have a moderate level of comparability, considering the existence of controls for additional factors. Table 2 summarizes the assessment of quality for the included studies in this meta-analysis.

Table 2

Risk of bias summary: review authors' judgement about the risk of bias for items in included studies

\begin{tabular}{|c|c|c|c|c|c|c|c|c|c|}
\hline $\begin{array}{l}\text { Study } \\
\text { (year) }\end{array}$ & $\begin{array}{l}\text { Representativeness } \\
\text { of the patients }\end{array}$ & $\begin{array}{l}\text { Selection } \\
\text { of non- } \\
\text { exposed } \\
\text { cohort }\end{array}$ & $\begin{array}{l}\text { Ascertainment } \\
\text { of } \\
\text { exposure }\end{array}$ & $\begin{array}{l}\text { Interest } \\
\text { outcome } \\
\text { not } \\
\text { present } \\
\text { at } \\
\text { start of } \\
\text { study }\end{array}$ & $\begin{array}{l}\text { Comparability } \\
\text { of } \\
\text { cohorts }\end{array}$ & $\begin{array}{l}\text { Control } \\
\text { for any } \\
\text { additional } \\
\text { factor }\end{array}$ & $\begin{array}{l}\text { Assessment } \\
\text { of } \\
\text { outcome }\end{array}$ & $\begin{array}{l}\text { Sufficiency } \\
\text { of } \\
\text { follow-up }\end{array}$ & $\begin{array}{l}\text { Adequacy } \\
\text { of } \\
\text { follow-up }\end{array}$ \\
\hline $\begin{array}{l}\text { Niinimäki } \\
\text { et al. } \\
2011\end{array}$ & - & - & - & - & - & + & + & - & - \\
\hline $\begin{array}{l}\text { Pandit et } \\
\text { al. } 2011\end{array}$ & - & - & - & - & - & + & - & - & - \\
\hline $\begin{array}{l}\text { Maier et } \\
\text { al. } 2015\end{array}$ & - & - & - & - & - & + & + & - & - \\
\hline $\begin{array}{l}\text { Berend et } \\
\text { al. } 2017\end{array}$ & - & - & - & - & - & + & - & - & + \\
\hline $\begin{array}{l}\text { Hamilton } \\
\text { et al. } \\
2017\end{array}$ & - & - & - & - & - & + & + & - & + \\
\hline -, low risk & bias; + , high risk of $b$ & & & & & & & & \\
\hline
\end{tabular}

\subsection{OKS}

Three studies compared postoperative OKS in the PTCL and FTCL UKA groups after following-up for $\geq 2$ years [15-17]. The pooled mean difference in postoperative OKS was $-2.92\left(95 \% \mathrm{Cl}-5.29\right.$ to $-0.55 ; \mathrm{P}=0.02 ; \mathrm{I}^{2}=53 \%$, Fig. 2), indicating that postoperative OKS was significantly higher by 2.92 in FTCL patients than in PTCL ones.

\subsection{AKS}

AKS-knee scores and AKS-function scores were assessed by two studies in groups of patients who underwent UKA with PTCL and FTCL, at a mean follow-up of $>3.9$ years $[15,17]$. The pooled mean difference in AKS-knee scores was $-2.90\left(95 \% \mathrm{Cl}-9.14\right.$ to $-3.34 ; \mathrm{P}=0.36 ; \mathrm{I}^{2}=67 \%$, Fig. 3$)$, indicating 
that the pooled mean difference of postoperative AKS-knee scores between the two studies was similar in the two groups. In contrast, the pooled mean difference of postoperative AKS-function scores was 5.63 higher in the FTCL group than in the PTCL group $\left(95 \% \mathrm{Cl}-9.27\right.$ to $-1.98 ; \mathrm{P}=0.002 ; \mathrm{I}^{2}=0 \%$, Fig. 4).

\subsection{Reoperation Rate and Revision Surgery}

All five studies presented data on reoperation rates, which included arthroscopy, replacement of meniscal bearing, revisions to total knee arthroplasty (TKA), and open exploration [13-17]. Consequently, 39 reoperations were reported for 613 knees, among which were 21 (9\%) for 233 knees with PTCL and $18(4.7 \%)$ for 380 FTCL knees, respectively. The pooled results showed that the reoperation rate was statistically higher in patients who underwent UKA with PTCL than in patients who underwent UKA with FTCL $\left(21 / 233\right.$ vs. $18 / 380$, OR 2.24, 95\%Cl 1.15-4.38; n.s.; I $^{2}=0 \%$, Fig. 5).

Although the distributed revision cases were not available in the retrospective study by Niinimäki et al. [14], the other four studies reported 12 revision surgeries (nine for PTCL knees and two for FTCL knees). In the PTCL group, Maier reported five revisions, including one exchange of polyethylene inlay because of early infection (21 days), one TKA because of infection (44 weeks), one TKA after clinically and radiologically detected loosening with associated pain (12 months), and two arthroscopic procedures for associated pain (12 and 13 months each) [17]. Hamilton et al. reported four revisions at a mean of 5.9 years (range: 0.9 to 10.3 years): two were for progression of OA, one involving the addition of a lateral UKA (6.3 years), and one involving revision to a TKA (10.3 years); one revision was performed for loosening of the femoral component (7.2 years); and one was for persistent pain (0.9 years), both converted to TKA [15]. The FTCL group reported three revisions in total, including one open revision because of fixed Redon drainage (3 days), one TKA because of loosening of the femoral component (7.9 years), and one TKA for arthrofibrosis.

\subsection{Change in OKS}

Based on the data for preoperative and postoperative OKS available in three studies [15-17], we compared the gain in OKS between the PTCL and FTCL UKA groups after following-up for $\geq 2$ years. The pooled mean difference in the change in OKS was $-2.69\left(95 \% \mathrm{Cl}-4.79\right.$ to $-0.60 ; \mathrm{P}=0.01 ; \mathrm{I}^{2}=50 \%$, Fig. 6), indicating that the gain in OKS was significantly higher by 2.69 in the FTCL patients than in the PTCL patients.

\section{Discussion}

According to our search results, no similar meta-analysis has been conducted to compare the outcomes of patients with PTCL and FTCL following UKA. The main finding of this meta-analysis was that the post-UKA OKS and AKS-function scores were significantly higher in the FTCL patients than in the PTCL patients. The patients with FTCL appeared to benefit more from the UKA procedure than did the PTCL patients according to the change in the OKS. The pooled mean difference of postoperative AKS-knee scores in the FTCL group was also higher than in the PTCL group, but the difference was not statistically significant. However, the reoperation rate was higher in the patients who underwent UKA with PTCL than in the patients who underwent FTCL. Generally, the analysis showed that the postoperative patient-reported outcomes were superior, and the reoperation rate was lower in the patients with FTCL than in the patients with PTCL.

Goodfellow thought that the concept of no "bone-to-bone" abrasion (FTCL) in the medial compartment of the knee was a contradiction, so he recommended that orthopedists evaluate the cartilage loss thickness in the medial compartment of the knee by taking varus-stressed radiographs. If the varus-stressed radiographs failed to demonstrate "bone-to-bone" abrasion between the media femoral condyle and medial tibial plateau, UKA should not be performed $[7,8]$. The results of this meta-analysis supported that view. Generally, we concluded that patients with PTCL are not equally suitable for treatment with UKA as patients with bone on bone, which was consistent with what Hamilton et al. and Maier et al. concluded [15, 17].

The worse patient-reported outcomes and higher failure rates in patients with PTCL may be due to patient-related factors or the indication for UKA [16]. We assumed that patients who sought operative treatment with only partial cartilage damage had lower levels of tolerance to pain and higher expectations for postoperative outcomes $[16,18]$, which may have led to the differences in postoperative recovery. Another possible explanation for the worse outcomes and higher failure rates of patients with PTCL is that the cause of knee pain in the early phase of OA was different from that in the endstage [19]. We believe that merely thinning of the cartilage and surface fibrillation with marginal osteophytes is not a reliable explanation for disabling pain. Synovial inflammation and the release of biological mediators instead of cartilage damage could primarily explain why pain is associated with PTCL [20]. However, severe KOA mechanical factors are a much more significant source of pain [21-23]. If FTCL in the medial compartment of the knee cannot be proved, we should consider other causes for the persistent pain (for example, spine pathology, hip OA, or rheumatics) [24].

Another way to think about this is that we should only consider anteromedial OA as the probable cause of persistent intolerable knee pain if the cartilage buffering ability between the media femoral condyle and medial tibial plateau lessens significantly. Then, our UKA, which was designed to treat end-stage KOA and SONK, should be performed and would be expected to provide a superior outcome. When performing UKA in patients with PTCL, we may not remove the source of knee pain and consequently achieve an unacceptable outcome, which may necessitate one or more reoperations [15]. In fact, the main reason for reoperation reported in the five included studies was unresolved knee pain $[13,15,17]$. Logically, if a patient's main complaint (pain in the majority) is not resolved, they may ask for further treatment (reoperation). That is also the reason the FTCL group had higher OKS and AKS-knee scores, although the differences were not significant. Furthermore, alleviation of knee pain undoubtedly will accelerate the recovery of knee function and a return to physical activity $[25,26]$, which would be reflected by a higher AKS-function score in FTCL patients. 
Historically, the indications of UKA have always been developed with some controversy [7, 27]. Although FTCL of the medial compartment of the knee has been widely accepted as one of the indications of UKA [5, 6], some orthopedists believe that this kind of operation can achieve good outcomes in patients with PTCL as those in patients with FTCL [14]. To settle the controversy, researchers have come to different conclusions from studies with different designs [13-17]. Our meta-analysis indicated that the differences between the outcomes of the two groups were generally significant, which suggested to us that it is better to perform UKA in patients with "bone-to-bone" abrasions, which is the end-stage of KOA. Based on the results of this meta-analysis, we tend to recommend other kinds of operations such as high tibial osteotomy instead of UKA to treat patients with PTCL.

There were certain inevitable limitations of this study that should be considered. A common limitation to all meta-analyses, including ours, is that some studies are overlooked. To avoid this problem, we performed an extensive search with sensitive keywords and synonyms in multiple comprehensive databases and used the expertise of a clinical librarian. Another limitation is that all of the studies included in this meta-analysis were cohort studies, which will inevitably inherit the defects of observational comparison studies and result in some inherent heterogeneity because of uncontrolled bias [28]. In addition, the heterogeneity among studies may have been due to slight differences in other confounding factors, such as BMI, follow-up time, and diagnostic methods of FTCL and PTCL. Although systematic reviews with a meta-analysis are usually considered to be "high-quality evidence," we believe that our findings are, at most, of moderate quality when considering the limitations above. According to the outcome measures, many patientreported questionnaires, such as OKS and AKS, were used. Because of the high dependency on the patients' abilities to describe their daily activities several years earlier, this type of research is prone to "recall bias." Moreover, most of the descriptions of outcomes were not and probably could not be validated.

\section{Conclusion}

Our systematic review and meta-analysis showed that when compared with PTCL patients, patients with FTCL who underwent UKA achieved superior postoperative patient-reported outcomes and lower reoperation rates. We do not think that patients with PTCL in the medial compartment of the knee should be an indication for UKA. However, because too few studies can be included, the limited comparability of the studies available and insufficient accounting for confounding factors in most studies, the overall quality of the included studies was limited. In the future, more high-quality studies are needed to confirm the results of this meta-analysis.

\section{Abbreviations}

\section{UKA}

unicompartmental knee arthroplasty

FTCL

low back painfull-thickness cartilage loss

PTCL

partial-thickness cartilage loss

CNKI

China National Knowledge Infrastructure

OKS

Oxford Knee Score

AKS

American Knee Society

NOQAS

Newcastle-Ottawa Quality Assessment Scale Table

BMI

body mass index

\section{Declarations}

\section{Ethics approval and consent to participate}

Not applicable

\section{Consent for publication}

All the authors approved the manuscript for publication.

\section{Availability of data and material}

All data generated and analyzed during the study are available from the corresponding author upon request.

\section{Competing interests}

The authors declare that they have no conflict of interests. 


\section{Funding}

Not applicable

\section{Authors' contributions}

BLW and WGW conceived and designed the study. JHM and YY searched and selected relevant studies. BLW and WGW extracted and interpreted data. JHM and YY analyzed the data. JHM and YY wrote the paper. DBY and WSG critically reviewed and approved the final manuscript.

\section{Acknowledgments}

This project was supported by grants from the National Natural Science Foundation of China (No. 81772350), Beijing Municipal Science and Technology Commission (No. Z171100001017209), National Key Research and Development Program of China (No. 2017YFC0108102), Beijing Natural Science Foundation区No. 7204301囚and China-Japan Friendship Hospital project (No. 2018-1-QN-9).

\section{References}

1. Hunter DJ. and S. Bierma-Zeinstra(2019)Osteoarthritis. Lancet 393(10182):1745-1759.

2. Martel-Pelletier J, et al. Osteoarthritis Nat Rev Dis Primers. 2016;2:160-72.

3. London NJ, Miller LE. and J.E. Block(2011)Clinical and economic consequences of the treatment gap in knee osteoarthritis management. Med Hypotheses 76(6):887-892.

4. Willis-Owen CA, et al.(2009)Unicondylar knee arthroplasty in the UK National Health Service: an analysis of candidacy, outcome and cost efficacy. Knee 16(6):473-478.

5. Price AJ, et al.(2004)Sagittal plane kinematics of a mobile-bearing unicompartmental knee arthroplasty at 10 years: a comparative in vivo fluoroscopic analysis. J Arthroplasty 19(5):590-597.

6. Rodriguez-Merchan EC. and P. Gomez-Cardero(2018)Unicompartmental knee arthroplasty: Current indications, technical issues and results. EFORT Open Rev 3(6):363-373.

7. Tyagi V. and M. Farooq(2017)Unicompartmental Knee Arthroplasty: Indications, Outcomes, and Complications. Conn Med 81(2):87-90.

8. Murray DW, Goodfellow JW. and J.J. O'Connor(1998)The Oxford medial unicompartmental arthroplasty: a ten-year survival study. J Bone Joint Surg $\operatorname{Br} 80(6): 983-989$.

9. Goodfellow J, O'Connor J. and D.W. Murray(2002)The Oxford meniscal unicompartmental knee. J Knee Surg 15(4):240-246.

10. Berend KR, Lombardi AJ. and C.A. Jacobs(2017)The Combination of Preoperative Bone Marrow Lesions and Partial-Thickness Cartilage Loss Did Not Result in Inferior Outcomes After Medial Unicompartmental Knee Arthroplasty. J Arthroplasty 32(10):3000-3003.

11. Wells G, Shea B, O'Connell D, Peterson J, Welch V, Losos M, Tugwell. P(2013)The Newcastle-Ottawa Scale (NOS) for assessing the quality of nonrandomized studies in meta-analyses. .

12. Price AJ, Waite JC. and U. Svard(2005)Long-term clinical results of the medial Oxford unicompartmental knee arthroplasty. Clin Orthop Relat Res (435):171-180.

13. Mukherjee K, et al.(2008)The Oxford unicompartmental knee arthroplasty: a radiological perspective. Clin Radiol 63(10):1169-1176.

14. Niinimaki TT, et al.(2011)Unicompartmental knee arthroplasties implanted for osteoarthritis with partial loss of joint space have high re-operation rates. Knee 18(6):432-435.

15. Hamilton TW, et al.(2017)Unsatisfactory outcomes following unicompartmental knee arthroplasty in patients with partial thickness cartilage loss: a medium-term follow-up. Bone Joint J 99-B(4):475-482.

16. Pandit $\mathrm{H}$, et al.(2011)Unicompartmental knee replacement for patients with partial thickness cartilage loss in the affected compartment. Knee 18(3):168-171.

17. Maier MW, et al.(2015)Unicompartmental knee arthroplasty in patients with full versus partial thickness cartilage loss (PTCL): equal in clinical outcome but with higher reoperation rate for patients with PTCL. Arch Orthop Trauma Surg 135(8):1169-1175.

18. Fingleton C, et al.(2015)Pain sensitization in people with knee osteoarthritis: a systematic review and meta-analysis. Osteoarthritis Cartilage 23(7):1043-1056.

19. Emery IH. and G. Meachim(1973)Surface morphology and topography of patello-femoral cartilage fibrillation in Liverpool necropsies. J Anat 116 (Pt 1):103-120.

20. Goldring MB. and K.B. Marcu(2009)Cartilage homeostasis in health and rheumatic diseases. Arthritis Res Ther 11(3):224.

21. Kean WF, Kean R, Buchanan WW.(2004)Osteoarthritis: symptoms, signs and source of pain. Inflammopharmacology 12(1):3-31.

22. Pan F, et al.(2017)The association of knee structural pathology with pain at the knee is modified by pain at other sites in those with knee osteoarthritis. Clin Rheumatol 36(11):2549-2555.

23. Arendt-Nielsen L, et al.(2015)A mechanism-based pain sensitivity index to characterize knee osteoarthritis patients with different disease stages and pain levels. Eur J Pain 19(10):1406-1417. 
24. Goodfellow JW, et al.(1988)The Oxford Knee for unicompartmental osteoarthritis. The first 103 cases. J Bone Joint Surg Br 70(5):692-701.

25. Berninger MT, et al.(2018)Effect of local infiltration analgesia, peripheral nerve blocks, general and spinal anesthesia on early functional recovery and pain control in unicompartmental knee arthroplasty. BMC Musculoskelet Disord 19(1):249.

26. Witjes S, et al.(2016)Return to Sports and Physical Activity After Total and Unicondylar Knee Arthroplasty: A Systematic Review and Meta-Analysis. Sports Med 46(2):269-292.

27. Kozinn SC, Scott R. Unicondylar knee arthroplasty. Journal of Bone Joint Surgery American Volume. 1989;71(1):145-50.

28. Klebanoff MA. and J.M. Snowden(2018)Historical (retrospective) cohort studies and other epidemiologic study designs in perinatal research. Am J Obstet Gynecol 219(5):447-450.

\section{Figures}
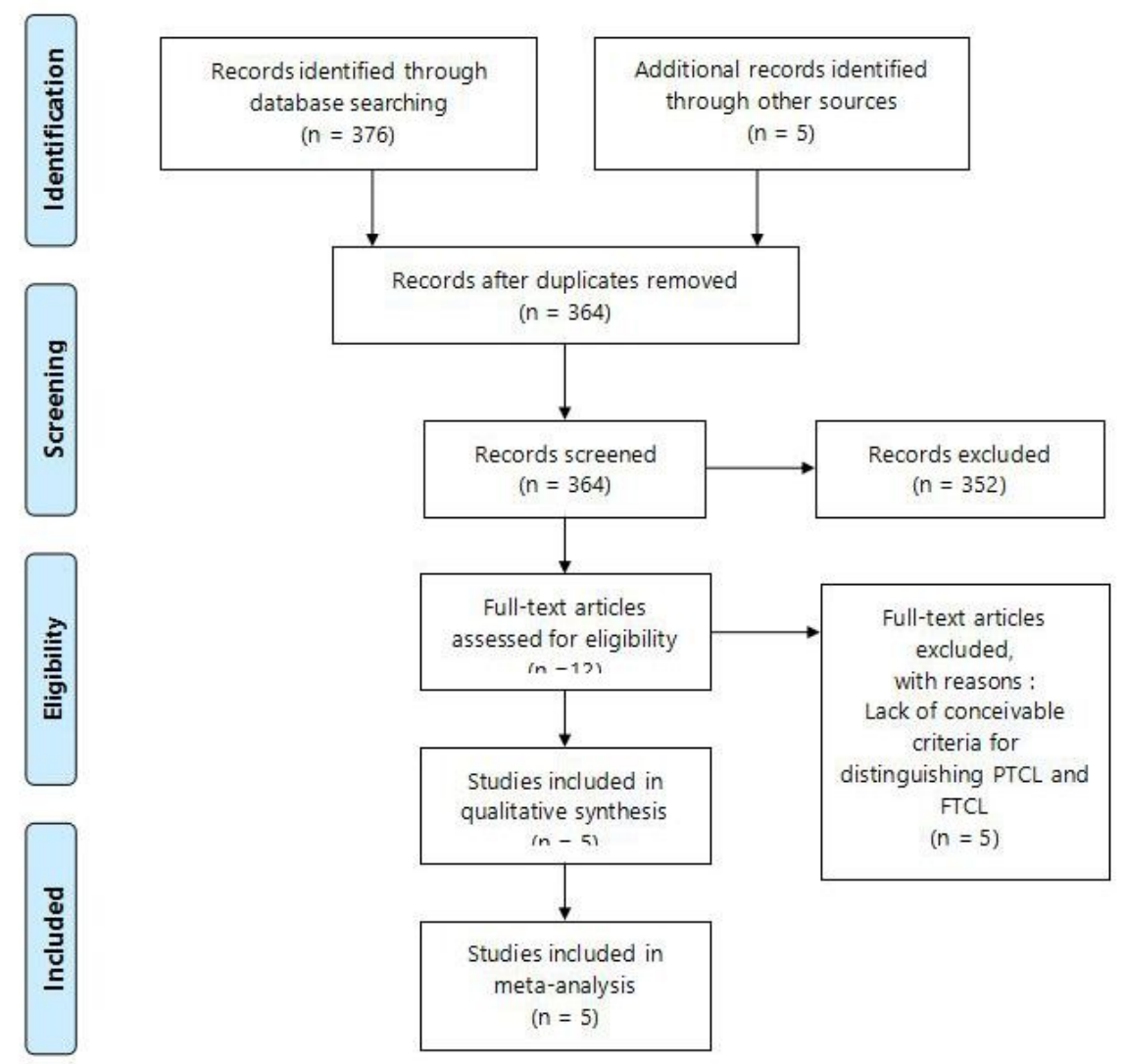

Figure 1

Flow chart of the literature search. FTCL indicates full-thickness cartilage loss. PTCL indicates partial-thickness cartilage loss.

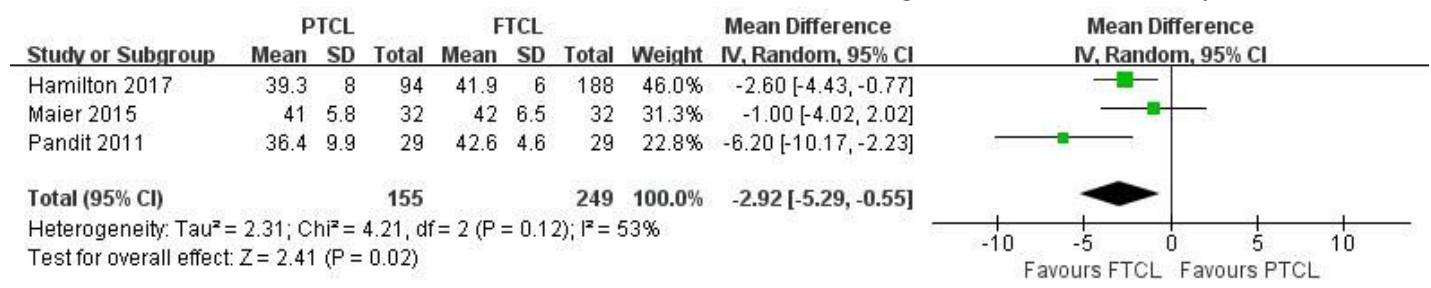

\section{Figure 2}

Forest plots of studies comparing postoperative OKS scores between PTCL and FTCL groups. 
PTCL FTCL $\quad$ Mean Difference Mean Difference

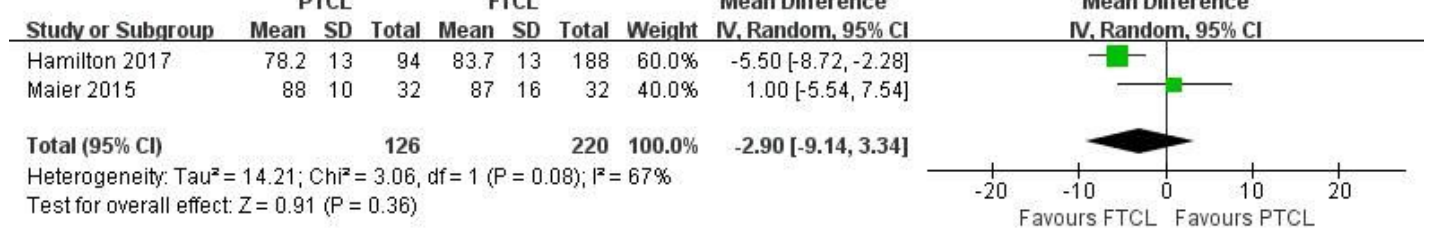

\section{Figure 3}

Forest plots of studies comparing postoperative AKS-knee scores between PTCL and FTCL groups.

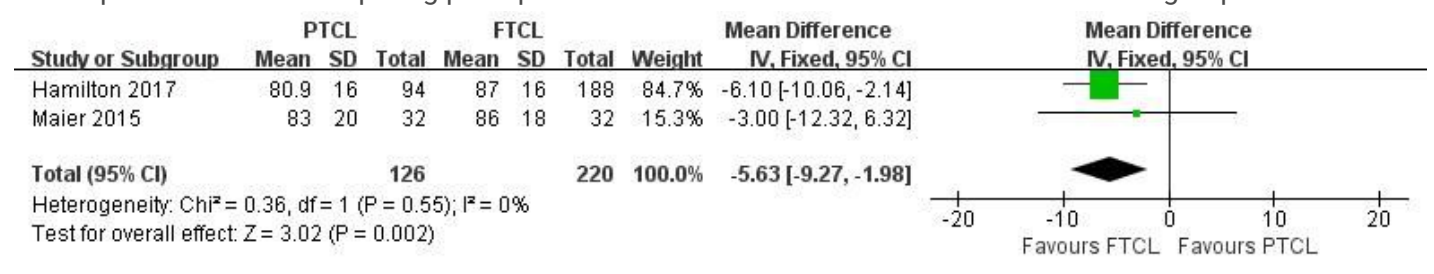

\section{Figure 4}

Forest plots of studies comparing postoperative AKS-function scores between PTCL and FTCL groups.

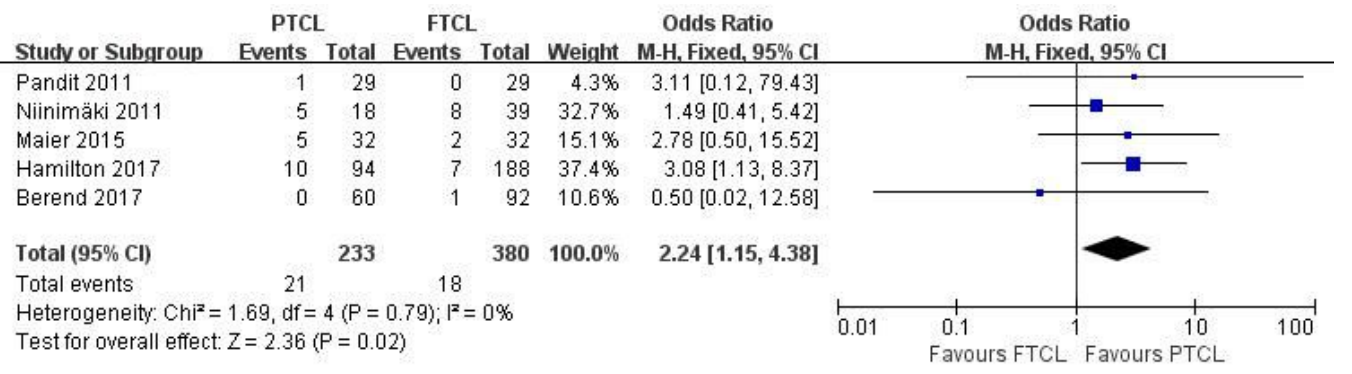

\section{Figure 5}

Forest plots of studies comparing reoperation rate between PTCL and FTCL groups.

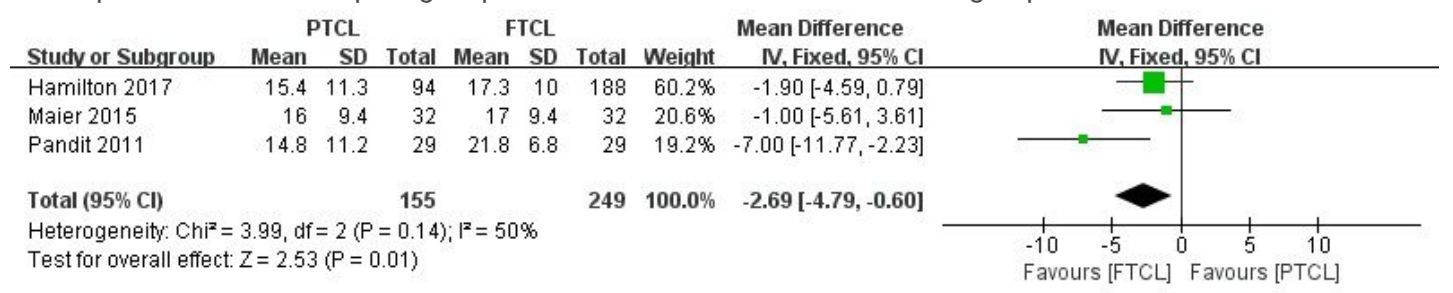

\section{Figure 6}

Forest plots of studies comparing the change in OKS between PTCL and FTCL groups. 